\title{
Finding K Shortest Paths in a Network Using Genetic Algorithm
}

\author{
Meenakshi Moza \\ MRIIRS/FET/CSE/Faridabad,Haryana,121003 \\ E-mail: meenakshi.fet@mriu.edu.in \\ Suresh Kumar \\ MRIIRS/FET/CSE/Faridabad,Haryana, 12100 \\ E-mail: sureshkumar.fet@mriu.edu.in
}

Received: 28 November 2019; Accepted: 30 March 2020; Published: 08 October 2020

\begin{abstract}
With the advent of new applications, different service needs come up. These needs could be in the form of reliability in delivering data, capacity amount in a particular range and certain amount of permissible delay. In order to provide high Quality of service to Networks, it is essential to provide a path between a given source and multiple destinations which satisfy certain constraints. For a domain catering to high QoS, there is a request of resources with certain constraints by all the applications. Speed and Scalability which are not flexible in terms of Network size and Topology are the basic issues to be considered here. Multimedia applications in general make use of $\mathrm{k}$ shortest paths whenever communication is to be carried out between a single source and one or more than one destination. In this paper, a genetic algorithm is used, which helps in determination of $\mathrm{k}$ shortest paths from a source node to more than one destination node, with bandwidth constraint. The algorithm makes use of the connection matrix as well as link bandwidth for determination of $\mathrm{k}$ shortest paths. The significance of using $\mathrm{K}$ shortest paths in a network is to increase Throughput and Packet delivery ratio.
\end{abstract}

Index Terms: Routing Optimization, Quality of Service (QOS), Shortest path, Fitness, Genetic Algorithm (GA), Crossover, Mutation, Open Shortest Path First (OSPF), Bandwidth, Connection matrix.

\section{Introduction}

The least cost route from a source node to another node is the shortest path through a network. This is the normal route which is given, preference, between the two specified nodes. If the shortest path is not available because of congestion or link break or node failure, it becomes essential to find the second shortest path. This can be true if the destination node is the same or different as well. If the second path also becomes unavailable due to some reason, what is required is the third path. The paths which are derived in such a manner are called $\mathrm{k}$ shortest paths. Initially the shortest path between the two nodes needs to be computed, before it is possible to compute (k-1) shortest paths.

The application of $\mathrm{k}$ shortest paths is in the field of telecommunication, transportation, science and operational research units. The above introduction can be summarized by saying that, any two nodes in a network, can be connected by more than one path, which means that, one can find not only the shortest path between any two nodes, but also (k-1) other paths. The application of such a work is because of shortest path being the most congested one[6,9,10]. Another application is, using it to track multiple objects and designing a transit network that improves the experiences of passengers to travel in public means of transport.

Two variations of $K$ shortest paths can be taken. The first one comprises of a path being used where the same node is visited couple of times and the second one is to deal with simple loopless $\mathrm{K}$ shortest path routing. Here the second case is considered.

Open Shortest Path First (OSPF) helps us to find the shortest path between each source and destination[3,4,8]. The limitation here is that of the overloading, in the shortest path or link congestion occurring anywhere in between. The arrival of the packets at the desired destination with the delay or queuing on the way or router processing can result in service quality being affected[1,5,19]. Complexity of configuration, not capable of supporting unequal load balance and high memory requirement are some of the limitations of OSPF and Constrained Routing, Unequal Load distribution, and not able to handle multiple link and node failures are some of the limitations of Multiple Routing Configuration

All these limitations can be taken care of by optimization of IP network. Genetic Algorithm (GA), an evolutionary and optimization algorithm, is the answer to this. It puts forward a solution, to find paths which are good alternatives against the overloaded path between source and destination. 
The basic steps that comprise a genetic algorithm are:

1. Initialization: An initial population is created. The population is generated randomly and can be of any size, that is, from only a few individuals to thousands. The initial population is as shown in Fig.1 (To express population in terms of Chromosomes by the help of nodes).

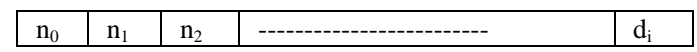

Fig.1. Initial population in terms of chromosomes

where $\mathrm{n}_{0}$ is the first node and $\mathrm{d}_{\mathrm{i}}(\mathrm{i}=0,1,2 .$.$) are the destination nodes.$

The purpose of Fig.1 is to represent population in terms of chromosomes by making use of nodes. (It makes it easier for a novice who is reading my paper to understand how an initial population is created. Actually i wanted to go stepwise for a novice otherwise many times it happens we do not understand how a person starts his/her research work implementation).

2. Evaluation: The members of the population are then evalu ated and a 'fitness' for that individual is calculated.

How well the individual fits in with the desired requirements helps to calculate the fitness value. Here the shortest source to destination path should satisfy the condition.

3. Selection: Improving the overall fitness of the population has to be the final goal. By means of selection, the bad designs are discarded and the best individuals in the population are kept back. Individuals with better fitness values to be selected for the next generation, has to be the basic idea.

4. Crossover: New individuals are created by the combination of aspects of selected individuals. By combination of certain traits from two or more individuals, a better offspring is created which will inherit the best of traits from both the parents.

5. Mutation - A little bit of randomness, needs to be added to the population genetics. This is essential so that combination of solutions created would be in our initial population. To an Individual's genome, small changes done at random is called mutation.

6. After the second generation is obtained, step 2 is started again till step 5 is reached.

Crossover and mutation are the power behind genetic algorithms. Exchanging bits from parents are used to form offspring, with the condition that any good solution will give rise to better ones. Crossover operation will always occur with a probability called as the crossover probability or crossover rate $\left(\mathrm{p}_{\mathrm{c}}\right)$. If the candidates or individuals do not go through crossover, the result is unmodified individuals. The operator of mutation is responsible for bringing about a change in some portions in candidates with a probability called mutation probability or mutation rate ( $\mathrm{pm}_{\mathrm{m}}$ ) Genetic algorithm's performance and behavior get affected by selection of $\mathrm{p}_{\mathrm{c}}$ and $\mathrm{p}_{\mathrm{m}}$. Higher the value of crossover rate, the faster exploitation of GA, to reach local optima occurs. If the value of crossover probability is too large then, individuals would be disrupted faster than they are exploited and mutation probability controls genetic algorithm speed. The value of $\mathrm{p}_{\mathrm{c}}$ should be in the range $0.5 \sim 1.0$, and the value of $\mathrm{p}_{\mathrm{m}}$ should be in the range $0.001 \sim 0.05$ was derived after a lot of literature survey[2,12,13,14]

In this paper, Genetic Algorithm is applied for solving the problem of $\mathrm{K}$ shortest paths, based on network link bandwidth. The paper is organized as follows. Section Ia gives the Introduction to the subject, Ib talks about the Motivation behind this work and 1c tells us about the Contribution of the Paper. Section II talks about related works. Section III presents the detailed overview of network analysis using GA. Section IV comprises the result analysis and section $\mathrm{V}$ discusses the conclusions drawn and future scope.

\section{Motivation Behind this Work}

User requirements of Computer Networks are changing very fast. They want information anytime and anywhere. A lot of applications like audio, video conversation, video streaming, etc. are using the networks for transferring data. These types of applications have special requirements in the terms of reliability, bandwidth, jitter, delay, etc. The network should possess these qualities to satisfy the demands of the users. These qualities in the right proportion comprise Quality of Service (QoS). The importance of QoS, results in finding techniques to provide reliable network performance and at the same time utilization of network resources efficiently. Open shortest Path First (OSPF) is one such technique which is responsible for creating a topological map of the network. It gives a good network performance due to its improved efficiency and effectiveness of routing. However, Complexity of Configuration, inability to support unequal load balance and high memory requirement are some issues in OSPF. The issues in OSPF are removed in Multiple Routing Configuration (MRC) scheme[17,26,28]. The MRC Protocol is based on storing additional information pertaining to routing and the packets are forwarded immediately over already configured next hops, once the failure is detected. Constrained Traffic Routing, Improvement required in load distribution and MRC not able to handle multiple link and node failures are the issues seen in the MRC. Route Optimization is used to solve the issues of MRC. This helps in reducing the operational problems of the network in addition to giving an improved performance. Also, overloading, 
delay and queuing can be taken care of by network optimization using Genetic Algorithm. In order to provide high Quality of service to Networks, it is essential to provide a path between a given source and multiple destinations which satisfy certain constraints. Multimedia applications in general make use of $\mathrm{k}$ shortest paths whenever communication is to be carried out between a single source and one or more than one destination.

\section{Contribution of Paper}

A comparison is carried out between Open Shortest Path First(OSPF), Open Shortest Path First with Genetic Algorithm (OSPA) and OSPAK. It is seen from the graphical representation that Throughput and Packet Delivery Ratio is highest when OSPAK is used but OSPA gives lesser Packet loss and Delay. Usage of OSPA or OSPAK will therefore be Application specific, meaning thereby that it depends on the users priority with respect to Packet Delivery Ratio and Delay.The above mentioned points are the actual contribution of this paper.However We can summarize the usage of $\mathrm{K}$ shortest paths as below:

K shortest paths are used for the following reasons:

1. Add on Constraints- It is used to find a path which satisfies some constraints beyond small length.

2. Evaluation of the Model-Certain paths are used for modelling certain problems which have known solutions without taking into account formulation of the path.

3. Analysis of the sensitivity of the solution-When more than one path is computed, sensitivity of the optimal solution with respect to changing the parameters of the problem can be determined.

4. Generation of Alternatives-Examination of more than one optimal solution of a problem will result in giving a better understanding of the problem.

\section{Related work:}

Accuracy, Authority, Objectivity, Currency and Coverage are the five basic criteria for information evaluation from any source.

Gonen, B. \& Yuksel, M., (2011) analyze the routing operation taking place on the internet. In simple terms it can be expressed as follows: When data is sent from source to destination, the complete information is divided into chunks called as packets. The header information in the form of an IP address is attached to the packet. The data packets are then made to travel on the links towards the routers. The incoming data is then stored in queue before being processed by a particular router. It is the router which reads the IP address and determines the best way to forward the packet to its destination. The paper states that Genetic Algorithm (GA) is a problem solving search method which is a sum total of natural selection and genetics. The paper defines GA to be comprised of eight steps. Choosing the initial population is the first step. In the second, the fitness of each candidate is evaluated. The third step consists of applying the while do loop of termination condition not satisfying. The next step involves selection of the best candidate for reproduction. After this the next generation is bred through crossover and mutation. The sixth step is to do fitness evaluation for the offspring. The candidate showing worst ranking is replaced by their offsprings. After this the algorithm is ended. Bilal analyses the above algorithm for a network topology of 20 nodes and 62 links and finds a shortest path in a limited time.

Obeidat, A et.al. (2012) propose a new routing algorithm for networks based on a combination of GA and network delay analysis. The reason for using GA is that, it gives better solutions inspite of changes in network topology, i.e the addition or deletion of nodes or link and volume of the network. They further state that GA operations can be categorized into Coding operation, Initialization, Evaluation, Reproduction, Crossover, Mutation and Termination. Time constraint being the only problem with GA solutions, Obeidat states that the technique adopted is an approach which provides an optimal solution in less time. The literature review comprises of Munemoto's study which employs chromosomes of varying length for problem encoding. The next study was done by Inagahi who used chromosomes of fixed length. Here the chromosomes are integers in sequence and a node ID is represented by a gene. The node is selected from a group of nodes in a random fashion. In his methodology, he states that the configuration of the network is dependent on Multiparameter encoding scheme. From all the node combinations, a routing table is created. The various entries in the routing table correspond to the paths between node pairs.

Fadil, Y. (2010) states that evaluation of each path is done on the basis of cost (shortest path) to find the routes in most of routing algorithms. In case of overloading or congestion taking place in the shortest path, optimization based on other parameters needs to be carried out to get better solutions. Genetic Algorithm (GA) is an optimization algorithm and Fadil puts forward a genetic algorithm. The basic solution lies here in providing alternative paths instead of overloaded paths so that there is better utilization of network resources and thereby improved QOS. The chromosomes of varying length and their genes are used for encoding purposes. Crossover and mutation provide a searching facility giving an improvement in solution quality and increased speed of convergence. GA is a method of programming which is similar to evolution in biology. For a particular problem to be solved, the input to GA is a set of solutions for which encoding is done and a metric called fitness function is used for quantitative evaluation of each candidate. These 
candidates are basically solutions which are already working but by making use of GA, they are improving and normally they are randomly generated. Each candidate is evaluated according to the fitness function. In this randomly generated pool of candidates, deletion of those which are not working takes place. Some of the candidates show promise and are therefore kept by and allowed to reproduce. Multiple copies of the same are produced and during copying some random changes are introduced. The copies may not be hundred percent perfect. These offsprings are then made to go to the next generation, giving rise to a new pool of candidate solutions. Then again, they are evaluated through a second round of fitness. The candidates which do not get better are deleted, but because of randomly varying certain candidates of the population, some candidates show improvement. These particular candidates are chosen and copied over to the next generation. It is expected that the average value of fitness of the population increases for every round and once the process is repeated thousands of times, good solutions to the problem at hand are found.

Ahmed, Y. (2010) describes a genetic algorithm for finding $\mathrm{k}$ shortest paths from a single source to multiple destination nodes. He presents the algorithm in terms of the connection matrix of the network analyzed and makes use of link bandwidth for determination of k shortest paths. The algorithm is applied to two networks comprising of 8 nodes and 20 nodes respectively, and calculates the k shortest paths for each destination node in both cases. He further states that in order to find $\mathrm{K}$ shortest paths with bandwidth constraint, all the paths from source to destination have to satisfy the condition that the bandwidth of the requisite path has to be greater than or equal to user defined bandwidth. By plotting a graph between K shortest paths and mutation probability, Ahmed proves that the number of K shortest paths decrease with a decrease in mutation probability.

Sonam, J. \& Sandeep, S. (2012) discuss Mobile adhoc networks as being wireless networks, which are self organized and do not work on any fixed type of infrastructure. Their topology is time variant and therefore it is very challenging to develop routing techniques for Manets for data transmission and reception. For this reason GA is used as a tool for exploring data routing techniques in Manets. They further imply that GA needs to be used for the purpose of providing solutions for the dynamic problem of multicasting in Manets, for the solution for multicasting in Manets for energy efficient systems and also for the solution for optimization of routing in Adhoc networks. It can further be used for the solution for improvement in QOS selection of routes in Manets and lastly for Path versus bandwidth calculation in Manets which are TDMA based. They further summarize and say that bio inspired research should be used to find similarities or analogies between large biological systems and network models so that an improvement in design of Manets is carried out.

Kavitha, S. \& Nair, T., (2011) use particle swarm optimization (PSO) and genetic algorithm (GA) tool to calculate a path which is optimal in nature for routing in networks. Particle swarm optimization states that, when the birds fly together in a group, the bird position changes with change in the velocity of their flight and the flock of birds keep on updating their position depending on the bird who is leading the group. This concept is used for either function maximization or minimization by updating the velocity. This is analogous to cost of every iteration. The goal here is to find the particle nodes / paths which have the maximum cost (in terms of bandwidth) corresponding to the link of particle (path). In this approach the path where data can be sent depends on the fitness value obtained for the particle. Higher the fitness value, the less is the probability of data corruption during transmission of data. In the genetic algorithm approach, the fitness value of chromosomes is calculated using bandwidth itself. Here the network with nodes is assigned a priority value which is used for path encoding also. The construction of path takes place by beginning at the source and taking into account neighbor priorities. The neighbor having the maximum priority is chosen and the path is formed partially from source to this neighbor. This process takes place in continuation till destination node is reached. Simulation results show that both PSO and GA give the optimum path which is based on bandwidth. Twenty iterations were used and the hop counts were taken for both PSO and GA calculations. They gave quite similar range of value for convergence time. It was seen that improvement in results could be obtained by having more information on node environment.

Ahmed, Y. \& Hassan, M., (2015) analyze the path with minimum cost using genetic algorithm as a tool. The paper talks about an algorithm, where one or more constraints are to be satisfied to find a feasible path. The main goal here is to look for edges in such a way that it connects all those nodes, where the total edge cost from source to every node has a minimum value. Three sample networks of eight, eleven and sixteen nodes have been taken up in this paper. Analysis is done on the basis of connection matrix and cost matrix of the three networks. Search is carried out for the path of minimum cost which is constructed using the minimum cost path tree which is rooted at a particular node. The parameter values taken are population size equal to twenty, maximum generation equal to fifty, probability of crossover is equal to nine percent and probability of mutation is equal to 0.02 . For each of the networks, a set of chromosomes, shortest path set and cost has been tabulated. For an eight node network, there are seven shortest paths, sets, for a eleven node network, there are ten shortest paths, sets and for a sixteen node network, there are fifteen, shortest path sets. They further discuss that the same algorithm can be extended for the solution of a multi constrained path tree problem.

\section{Network Analysis Using GA}

Representation of a network is done by means of a graph $\mathrm{G}=(\mathrm{N}, \mathrm{L})$ where $\mathrm{N}$ represents a set of nodes and $\mathrm{L}$ stands for the links connecting the nodes. Let $Q=\left\{n_{0}, n i\right\}$ which is a subset of $N$ where $n_{0}$ is the source node and $n_{1}$ to $n_{i}$ is the set of destination nodes. Let $\mathrm{P}\left(\mathrm{n}_{0}, \mathrm{n}_{\mathrm{i}}\right)$ be a path from source $\mathrm{n}_{0}$ to destination node ni, where $\mathrm{i}=1$ to $\mathrm{n}$. This path possesses 
bandwidth characteristics.

The bandwidth of the path is $\mathrm{B}_{\mathrm{p}}=\operatorname{Min} \mathrm{B}_{1}$

where $\mathrm{l}_{\mathrm{i}}$ is the link between nodes ni and $\mathrm{ni}_{\mathrm{i}} \mathrm{l}$.

We have to find the path between source and destination where $B_{p}>=B_{G}$

Fig. 2 represents a sample 9 nodes network and Table 1 represents the k shortest paths for the 9 nodes Network.

Table 1. K shortest paths where source node is node 1 and destination (Des.)nodes are 2,4,5,8

\begin{tabular}{|c|c|c|c|}
\hline Des.Node & Shortest path & $\mathrm{K}$ & B.W(W) \\
\hline \multirow[t]{7}{*}{2} & 1,2 & 7 & 12 \\
\hline & $1,6,9,2$ & & 8 \\
\hline & $1,6,5,4,3,2$ & & 9 \\
\hline & $1,6,7,2$ & & 9 \\
\hline & $1,6,5,8,7,2$ & & 8 \\
\hline & $1,6,7,8,3.2$ & & 9 \\
\hline & $1,6,5,8,3,2$ & & 8 \\
\hline \multirow[t]{7}{*}{4} & $1,2,3,4$ & 7 & 12 \\
\hline & $1,6,5,4$ & & 9 \\
\hline & $1,2,7,8,4$ & & 8 \\
\hline & $1,2,9,6,5,4$ & & 8 \\
\hline & $1,2,7,8,5,4$ & & 8 \\
\hline & $1,6,7,8,4$ & & 8 \\
\hline & $1,6,5,8,4$ & & 8 \\
\hline \multirow[t]{8}{*}{5} & $1,6,5$ & 8 & 9 \\
\hline & $1,2,3,4,5$ & & 11 \\
\hline & $1,6,7,8,5$ & & 8 \\
\hline & $1,6,7,8,4,5$ & & 8 \\
\hline & $1,2,7,8,5$ & & 8 \\
\hline & $1,2,3,8,5$ & & 8 \\
\hline & $1,6,7,2,3,8,5$ & & 8 \\
\hline & $1,2,7,8,3,4,5$ & & 9 \\
\hline \multirow[t]{10}{*}{8} & $1,2,3,8$ & 10 & 11 \\
\hline & $1,2,7,8$ & & 9 \\
\hline & $1,2,9,6,5,8$ & & 8 \\
\hline & $1,2,9,6,7,8$ & & 8 \\
\hline & $1,6,5,8$ & & 8 \\
\hline & $1,6,7,8$ & & 9 \\
\hline & $1,6,5,4,8$ & & 8 \\
\hline & $1,2,7,6,5,8$ & & 8 \\
\hline & $1,6,9,2,7,8$ & & 8 \\
\hline & $1,6,9,2,3,8$ & & 8 \\
\hline
\end{tabular}




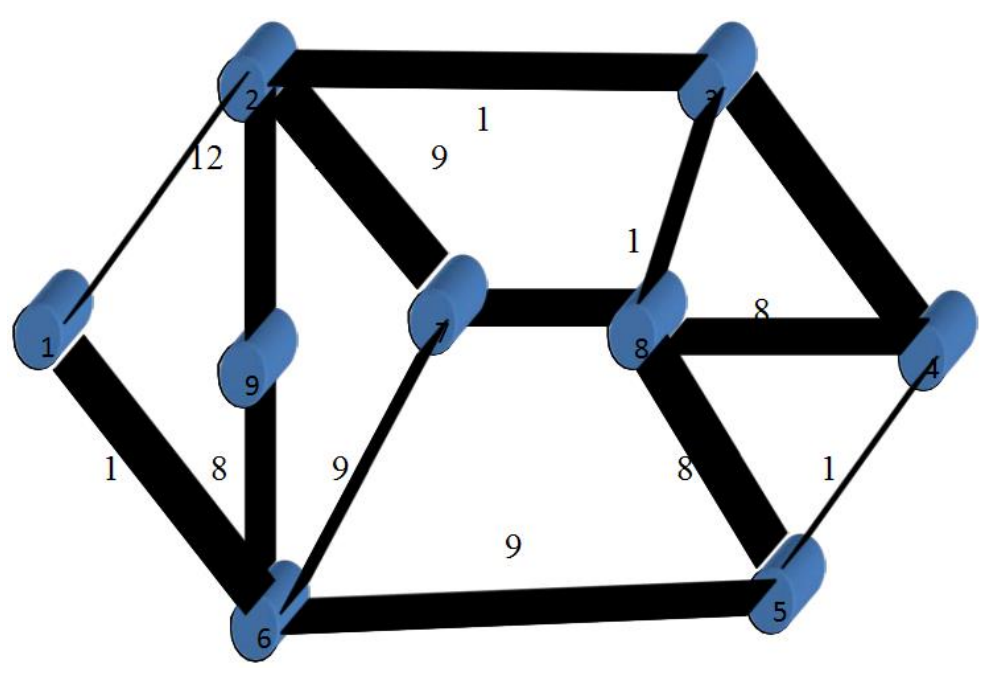

Fig.2. 9 Nodes Network

Fig. 3 represents another sample network of 22 nodes and Fig. 4 shows the connection matrix for the 22 nodes network.

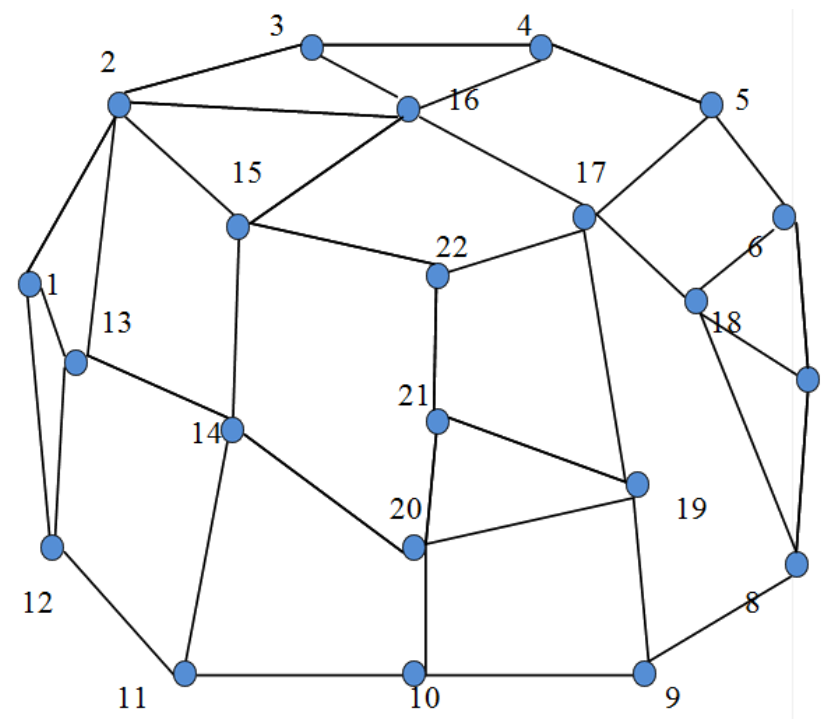

Fig.3. 22 Nodes sample network

\begin{tabular}{|c|c|c|c|c|c|c|c|c|c|c|c|}
\hline & 1 & 2 & 3 & 4 & 5 & 6 & 7 & 8 & 9 & - & 22 \\
\hline 1 & 0 & 1 & 0 & 0 & 0 & 0 & 0 & 0 & 0 & - & 0 \\
\hline 2 & 1 & 0 & 1 & 0 & 0 & 0 & 0 & 0 & 0 & - & 0 \\
\hline 3 & 0 & 1 & 0 & 1 & 0 & 0 & 0 & 0 & 0 & - & 0 \\
\hline 4 & 0 & 0 & 1 & 0 & 1 & 0 & 0 & 0 & 0 & - & 0 \\
\hline 5 & 0 & 0 & 0 & 1 & 0 & 1 & 0 & 0 & 0 & - & 0 \\
\hline 6 & 0 & 0 & 0 & 0 & 1 & 0 & 1 & 0 & 0 & - & 0 \\
\hline 7 & 0 & 0 & 0 & 0 & 0 & 1 & 0 & 1 & 0 & - & 0 \\
\hline 8 & 0 & 0 & 0 & 0 & 0 & 0 & 1 & 0 & 1 & - & 0 \\
\hline 9 & 0 & 0 & 0 & 0 & 0 & 0 & 0 & 1 & 0 & - & 0 \\
\hline- & - & - & - & - & - & - & - & - & - & - & - \\
\hline 22 & 0 & 0 & 0 & 0 & 0 & 0 & 0 & 0 & 0 & - & 0 \\
\hline
\end{tabular}

Fig.4. Connection matrix of 22 nodes

The connection matrix of 22 nodes shown above tells us, that the value is 0 whenever there is no direct link between two nodes and value is 1 when there is a direct link between the nodes. The sign " - in both the tables signifies that because of less space all the nodes have not been specified here. The values of 1 are replaced by the actual bandwidth in the Fig.5. 


\begin{tabular}{|c|c|c|c|c|c|c|c|c|c|c|c|}
\hline & 1 & 2 & 3 & 4 & 5 & 6 & 7 & 8 & 9 & ---- & 22 \\
\hline 1 & 0 & 5 & 0 & 0 & 0 & 0 & 0 & 0 & 0 & - & 0 \\
\hline 2 & 5 & 0 & 9 & 0 & 0 & 0 & 0 & 0 & 0 & - & 0 \\
\hline 3 & 0 & 9 & 0 & 5 & 0 & 0 & 0 & 0 & 0 & - & 0 \\
\hline 4 & 0 & 0 & 5 & 0 & 9 & 0 & 0 & 0 & 0 & - & 0 \\
\hline 5 & 0 & 0 & 0 & 9 & 0 & 8 & 0 & 0 & 0 & - & 0 \\
\hline 6 & 0 & 0 & 0 & 0 & 8 & 0 & 5 & 0 & 0 & - & 0 \\
\hline 7 & 0 & 0 & 0 & 0 & 0 & 5 & 0 & 9 & 0 & - & 0 \\
\hline 8 & 0 & 0 & 0 & 0 & 0 & 0 & 9 & 0 & 15 & - & 15 \\
\hline 9 & 0 & 0 & 0 & 0 & 0 & 0 & 0 & 15 & 0 & - & 0 \\
\hline-- & - & - & - & - & - & - & - & - & - & - & 0 \\
\hline 22 & 0 & 0 & 0 & 0 & 0 & 0 & 0 & 15 & 0 & - & 0 \\
\hline
\end{tabular}

Fig.5. 22 nodes network with bandwidth values

Table 2. K shortest paths where source node is node 1 and destination nodes are 8,12,15,18,20,22.

\begin{tabular}{|c|c|c|}
\hline Destination Node & Value of K & Bandwidth \\
\hline 8 & 10 & 9 \\
\hline 12 & 7 & 9 \\
\hline 15 & 6 & 9 \\
\hline 18 & 10 & 9 \\
\hline 20 & 7 & 9 \\
\hline 22 & 6 & 9 \\
\hline
\end{tabular}

The solution to the problem of finding K shortest paths for any sample Network is carried out by applying GA onto a initial population that is generated. Initially a population size is decided which gives us the number of chromosomes to be used for a particular population. When we talk about a chromosome, it is a list of nodes from a particular source to a particular destination. The steps for generation of initial population are as explained below:

1. Insertion of first node into the chromosome is carried out and this is the first gene. This gene has infinite bandwidth and zero delay.

2. Inclusion of a new node into the chromosome is carried out by looking for a link which starts at the last node in the chromosome.

3. In case of a link not being found, the chromosome is destroyed. This results in end of process and formation of a new chromosome.

4. In case of a presence of a link, random selection of one of the nodes connected to the last node of the chromosome is carried out. Insertion into the chromosome is done and removal of the link from the temporary link data base is carried out for prevention of loops.

5. Updation of the delay and bandwidth values is carried out.

6. Whenever the node that is inserted last is the destination node, process is ended otherwise repetition of the above process from step 2 takes place.

Once a chromosome is generated, the fitness value of that particular chromosome is calculated. It is the fitness value which confirms as to whether the particular chromosome is a candidate solution for that problem or not. In this scenario, the fitness value of the chromosome is calculated by taking bandwidth to be maximum and delay to be minimum in the population. The best chromosomes would be the one having the maximum value of bandwidth and minimum value of delay. In addition to this the maximum delay values and minimum bandwidth values of all the chromosomes present in the population are calculated. The chromosomes present in the population can be represented as a point $\mathrm{c}(\mathrm{u}, \mathrm{v})$ where $\mathrm{u}$ stands for delay and $\mathrm{v}$ stands for bandwidth of chromosome and $\mathrm{c}$ is calculation of the distance of a chromosome from the best chromosome gives the fitness function. The equation for the bandwidth and delay of chromosomes (Ch.) is as written below:

$$
\begin{gathered}
\text { Ch.B.W }\left(\mathrm{B}_{\mathrm{ch}}\right)=100-\left[\left(\mathrm{Max} \mathrm{B} \cdot \mathrm{W}-\mathrm{B}_{\mathrm{ch}) /(}(\mathrm{Max} \cdot \mathrm{B} \cdot \mathrm{W}-\mathrm{Min} . \mathrm{B} \cdot \mathrm{W})\right]^{*} 100\right. \\
\text { Ch.Delay }\left(\mathrm{D}_{\mathrm{ch}}\right)=100-\left[\left(\mathrm{D}_{\mathrm{ch}}-\mathrm{Min} . \mathrm{D}\right) /(\text { Max.D.-Min.D })\right]^{* 100}
\end{gathered}
$$

$$
\text { Therefore Fitness }=\mathrm{F}_{\mathrm{ch}}=\sqrt{\mathrm{B}_{\mathrm{ch}}}{ }^{2}+\mathrm{D}_{\mathrm{ch}}{ }^{2}
$$

When crossover is taking place, the chromosome with lower fitness is the first parent of the first couple. The other 
chromosomes are looked into in ascending order of fitness value which helps in finding chromosome that has a node common with first chromosome. The first and last nodes are common to all chromosomes and therefore not included in the search. The chromosome, which has a common node with first chromosome is the second parent of the first couple. The second best chromosome is one of the parents of the second couple and the search goes on for establishment of couples.

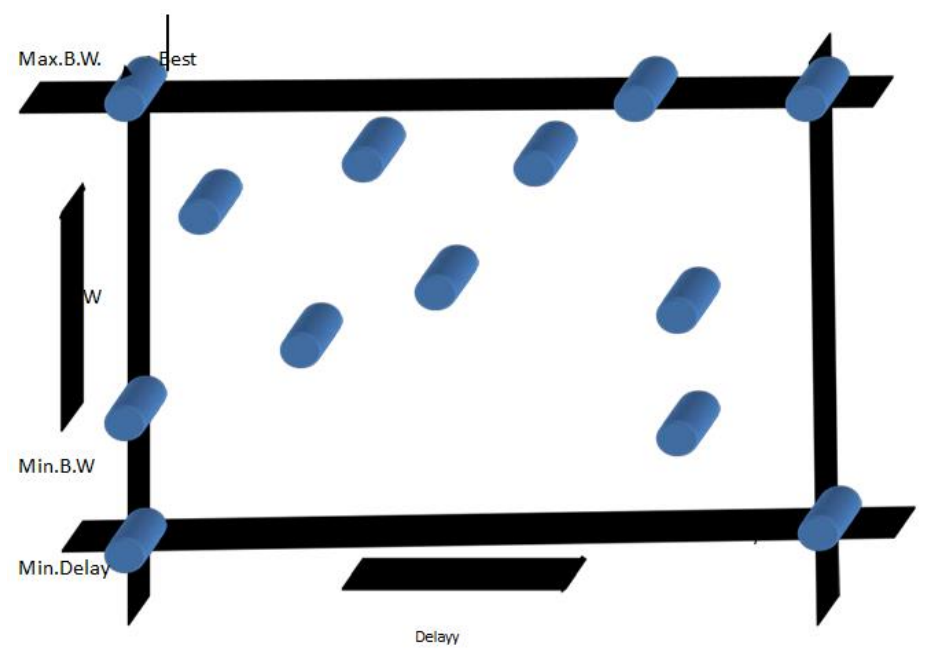

Fig.6. Best Chromosome

Consider the case of two chromosomes

CH1: 2-9-7-4-10-12-8-19-14

CH2: 2-7-6-11-5-13-15-17-10-16-14

The two chromosomes have two nodes 7 and 10 in common. Any one of these can be selected randomly as a crossover point. Node 10 say is selected as a crossover point. The new set of chromosomes after crossover is

NCH1:2-9-7-4-10-16-14

NCH2:2-7-6-11-5-13-15-17-12-8-19-14

Once crossover is complete, mutation is carried out by making use of mutation probability. Selection of a random node after excluding the first and last node and change in the subpaths which starts at the selected node comprises mutation. Once crossover and mutation is completed, there are two new chromosomes. Testing of chromosomes is carried out to test for looping which occurs in case of repetition of nodes in a path. Deletion of the chromosomes is carried out for all the nodes which come in between the repeating node and all repetitions of the repeating node.

This gives a path without loops. Addition of new chromosomes to the new generation is carried out. For all the new generation chromosomes, values of bandwidth and delay are calculated. It is also checked as to whether the new chromosomes are conforming to the constraints discussed earlier. The next step is to calculate the fitness values of the new chromosomes. There are two conditions and if either satisfies the algorithm shall terminate.

Condition 1:If a path is found which satisfies the above constraints.

Condition 2:After a particular number of iterations.

The inputs required to implement the algorithm for $\mathrm{K}$ shortest paths are listed below:

1. Population size ( Pop size)

2. Maximum generated population (Max. Gen.)

3. Probability of crossover $\left(\mathrm{pc}_{\mathrm{c}}\right)$

4. Probability of mutation $(\mathrm{pm})$

5. Source node $(\mathrm{n} 0)$

6. Destination node $\left(d_{0} \ldots \ldots . . d_{i}\right)$

The descriptive flow chart is as drawn: 


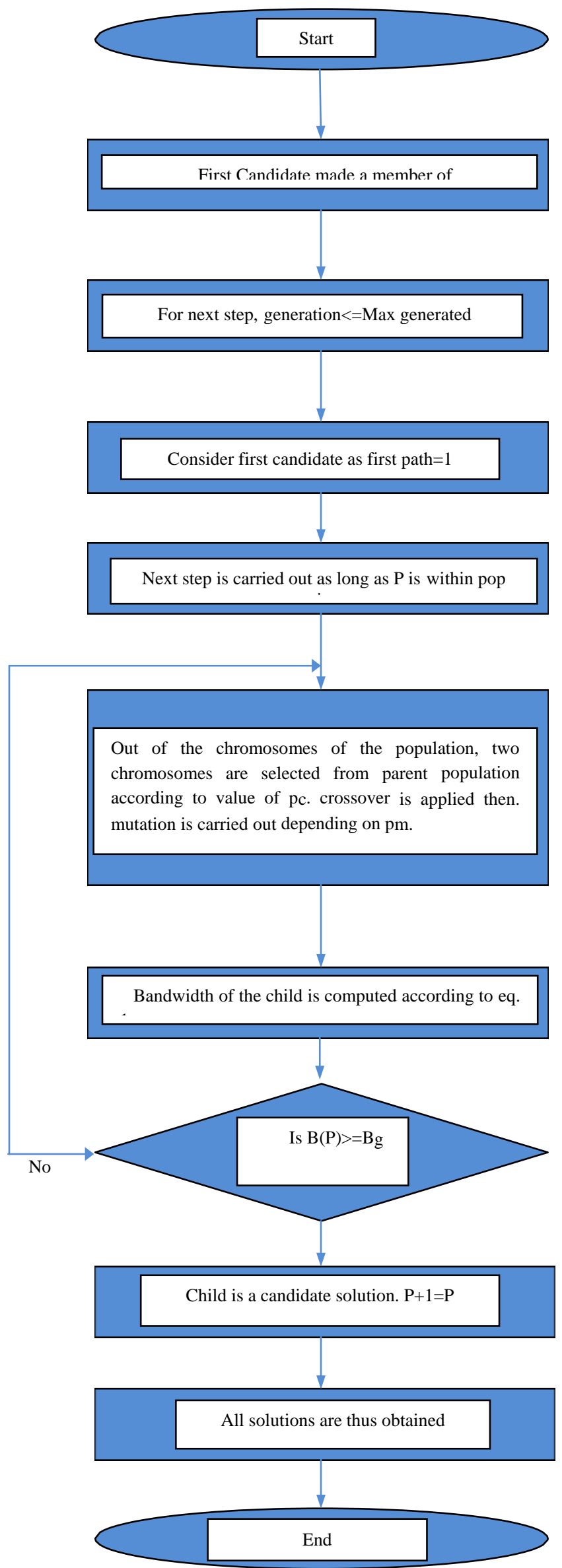

Fig.7. Flowchart for finding the $\mathrm{K}$ shortest paths 


\section{Result Analysis}

QoS is something, a flow seeks to attain in a network. Characteristics attributed to a flow are Reliability, Delay, Jitter and Bandwidth. Customer satisfaction is the main goal of providing a good quality of service. This is achieved by creating a balance between application demand and network performance. If application demand is high and network performance is low, no quality of service is obtained and if application demand is low and network performance is high, it results in wastage of resources. When the application demand is in sync with network performance, this results in high quality of service and it is the goal of a network design. As the number of users has increased, the size of internet has also increased and therefore care needs to be taken so that there is no degradation in performance of the network in terms of increase in delay, jitter and response time.

The main goal of a good QoS is providing priority, dedicated amount of bandwidth, controlled amount of jitter and latency requirement as per real time applications and an improvement in loss characteristics.

a) A comparison is carried out between Open Shortest Path First(OSPF), Open Shortest Path First with Genetic Algorithm (OSPA) and K shortest paths(OSPAK). The QoS parameter values namely Throughput, Packet delivery ratio, Packet loss, and Delay are as shown in Tables 3,4 5 and 6 . The Topology, shown in Fig. 8 has been used to study the performance of routing protocols OSPF, OSPA and OSPAK. The graphical comparison is shown in Figs. 9, 10, 11 and 12. The focus of the simulation is to study the effect of QoS parameters for OSPF, OSPA and OSPAK .

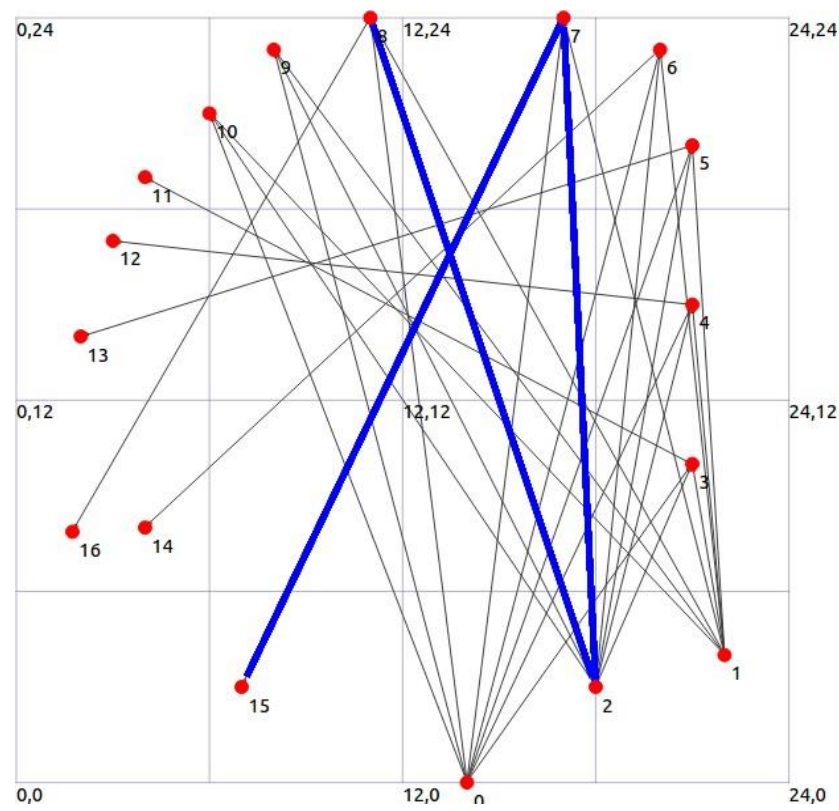

Fig.8. Topology Considered for Comparing OSPF,OSPA and OSPAK

Table 3. Throughput Comparison of Routing Protocols

\begin{tabular}{|c|c|c|c|}
\hline Packet size & OSPF & OSPA & K Shortest path \\
\hline 200 & 7481.25 & 16118.8 & 64493.8 \\
\hline 400 & 8028.34 & 24221.9 & 96925 \\
\hline 600 & 8242.5 & 24370.3 & 97518.8 \\
\hline 1000 & 8424.78 & 24359.4 & 97500 \\
\hline 1200 & 8471.28 & 24356.3 & 97500 \\
\hline
\end{tabular}

Table 4. Packet Delivery Ratio Comparison of Routing Protocols

\begin{tabular}{|c|c|c|c|}
\hline Packet size & OSPF & OSPA & K Shortest path \\
\hline 200 & 74.679 & 66.026 & 66.045 \\
\hline 400 & 85.384 & 99.219 & 99.257 \\
\hline 600 & 89.6 & 99.827 & 99.865 \\
\hline 1000 & 93.332 & 99.807 & 99.871 \\
\hline 1200 & 94.236 & 99.769 & 99.846 \\
\hline
\end{tabular}


Table 5. Packet Loss Comparison of Routing Protocols

\begin{tabular}{|c|c|c|c|}
\hline Packet size & OSPF & OSPA & K Shortest path \\
\hline 200 & 1424 & 5308 & 21220 \\
\hline 400 & 411 & 61 & 232 \\
\hline 600 & 195 & 9 & 28 \\
\hline 1000 & 75 & 6 & 16 \\
\hline 1200 & 54 & 6 & 16 \\
\hline
\end{tabular}

Table 6. Delay Comparison of Routing Protocols

\begin{tabular}{|c|c|c|c|}
\hline Packet size & OSPF & OSPA & K Shortest path \\
\hline 200 & 0.160204 & 0.012036 & 0.03494 \\
\hline 400 & 0.287409 & 0.02309 & 0.021176 \\
\hline 600 & 0.358253 & 0.008401 & 0.006304 \\
\hline 1000 & 0.298573 & 0.009677 & 0.007263 \\
\hline 1200 & 0.265277 & 0.010312 & 0.00774 \\
\hline
\end{tabular}

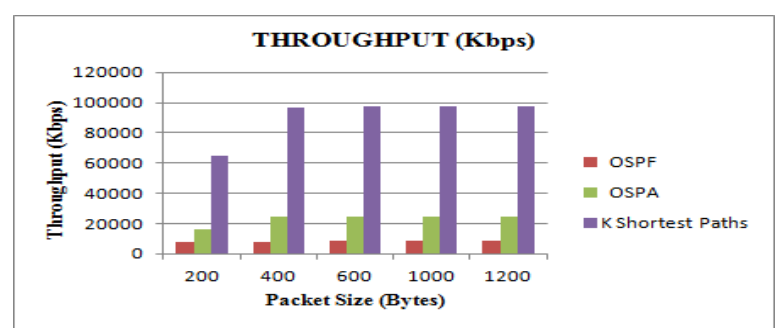

Fig.9. Packet Size vs Throughput for OSPF, OSPA, K Shortest Paths

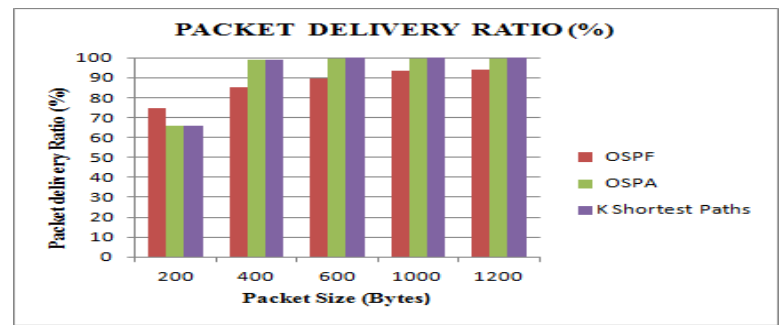

Fig.10. Packet Size vs Packet Delivery Ratio for OSPF, OSPA, K Shortest Paths

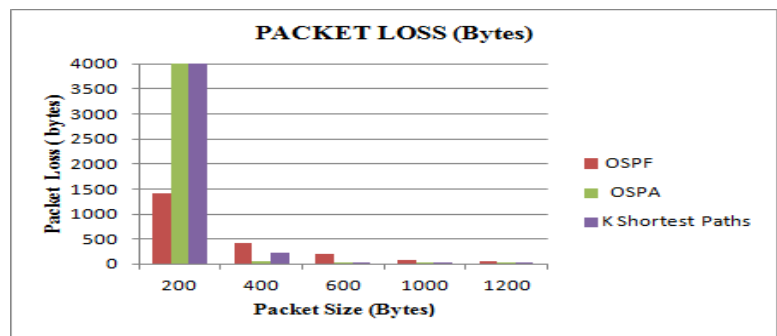

Fig.11. Packet Size vs Packet loss for OSPF, OSPA, K Shortest Paths

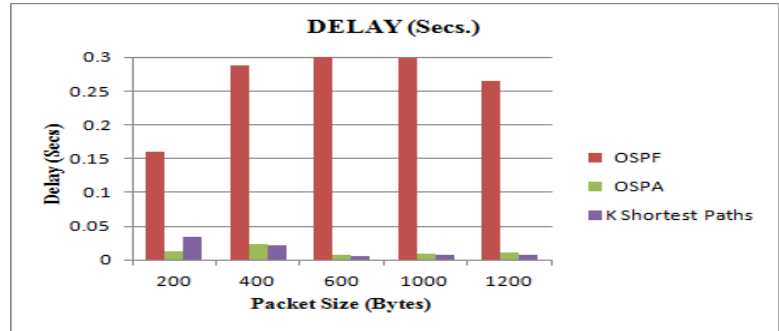

Fig.12. Packet Size vs Delay for OSPF, OSPA, K Shortest Paths 
It is seen from the graphical representation that Throughput and Packet Delivery Ratio is highest when K Shortest path Algorithm is used but OSPA gives lesser Packet loss and Delay. Both Throughput and Packet Delivery Ratio are directly proportional to received bytes of data. The received bytes being larger in K Shortest paths is the reason behind higher Throughput and higher Packet Delivery Ratio in K Shortest Path Algorithm. Usage of OSPA or K Shortest Path Algorithm will therefore be Application specific, meaning thereby that it depends on the users priority with respect to Packet Delivery Ratio and Delay.

b)Further Two scenarios namely Single source single destination (SSSD) and Multiple source multiple destination (MSMD) are considered as shown in Figs. 13a and 13b.

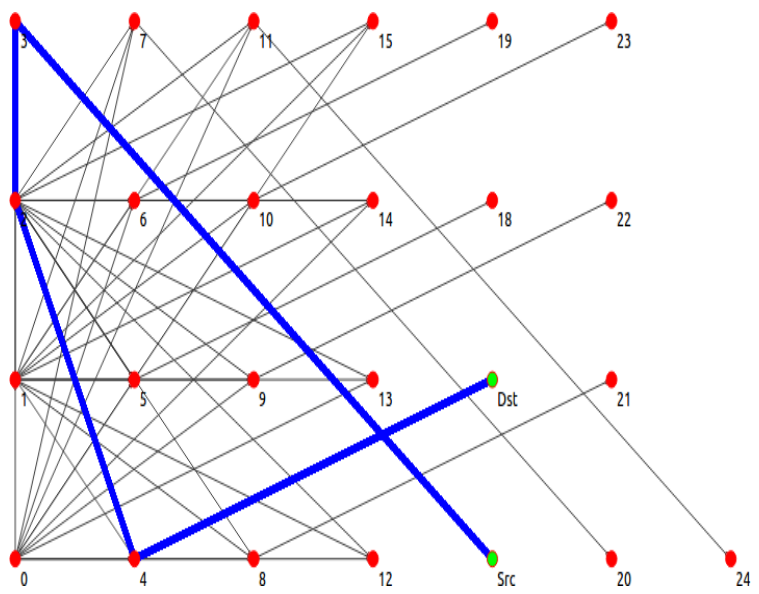

Fig.13a. Topology considered for SSSD

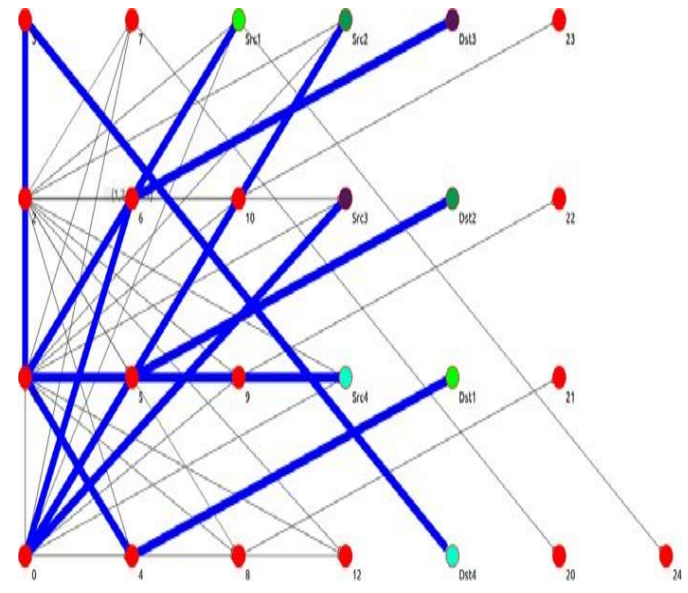

Fig.13b. Topology considered for MSMD

For the SSSD we have one source and one destination and for MSMD we have four sources, namely src1, src2, src3, src4 and four destinations namely dst1,dst2,dst3.dst4. QoS parameters, namely Throughput, Packet Delivery Ratio, Packet Loss and Delay are calculated for both the scenarios after application of Routing Algorithms Open Shortest Path Algorithm (OSPF), Optimized Shortest Path Algorithm (OSPA) and K Optimized Shortest Path Algorithm (OSPAK). The values for QoS parameters are tabulated as shown in Tables 7,8,9,10,11.12,13 and 14 and the graphs are plotted as shown in Figs. 14,15,16,17,18,19,20,21.

Table 7. SSSD Throughput

\begin{tabular}{|c|c|c|c|}
\hline Packet size & SSSD OSPF & SSSD OSPA & SSSD OSPAK \\
\hline 200 & 16118 & 13859 & 16118 \\
\hline 400 & 18734 & 19709 & 24221 \\
\hline 600 & 19767 & 22931 & 24370 \\
\hline 1000 & 20679 & 24359 & 24359 \\
\hline 1200 & 20915 & 24346 & 24356 \\
\hline
\end{tabular}


Table 8. MSMD Throughput

\begin{tabular}{|c|c|c|c|}
\hline Packet size & MSMD OSPF & MSMD OSPA & MSMD OSPAK \\
\hline 200 & 46545 & 55456 & 64493 \\
\hline 400 & 67971 & 78862 & 96925 \\
\hline 600 & 80872 & 91781 & 97518 \\
\hline 1000 & 86377 & 97468 & 97500 \\
\hline 1200 & 86551 & 97462 & 97500 \\
\hline
\end{tabular}

Table 9. SSSD Packet Delivery Ratio

\begin{tabular}{|c|c|c|c|}
\hline Packet size & SSSD OSPF & SSSD OSPA & SSSD OSPAK \\
\hline 200 & 52.73 & 56.77 & 66.03 \\
\hline 400 & 73.25 & 80.73 & 99.22 \\
\hline 600 & 84.17 & 93.93 & 99.83 \\
\hline 1000 & 95.56 & 99.81 & 99.81 \\
\hline 1200 & 98.91 & 99.73 & 99.77 \\
\hline
\end{tabular}

Table 10. MSMD Packet Delivery Ratio

\begin{tabular}{|c|c|c|c|}
\hline Packet size & MSMD OSPF & MSMD OSPA & MSMD OSPAK \\
\hline 200 & 26.37 & 56.79 & 66.05 \\
\hline 400 & 36.63 & 80.76 & 99.26 \\
\hline 600 & 42.09 & 93.99 & 99.87 \\
\hline 1000 & 47.79 & 99.84 & 99.87 \\
\hline 1200 & 49.46 & 99.81 & 99.85 \\
\hline
\end{tabular}

Table 11.SSSD Packet Loss

\begin{tabular}{|c|c|c|c|}
\hline Packet size & SSSD OSPF & SSSD OSPA & SSSD OSPAK \\
\hline 200 & 28068 & 6754 & 5308 \\
\hline 400 & 7941 & 1505 & 61 \\
\hline 600 & 3133 & 316 & 9 \\
\hline 1000 & 527 & 6 & 6 \\
\hline 1200 & 108 & 7 & 6 \\
\hline
\end{tabular}

Table 12. MSMD Packet Loss

\begin{tabular}{|c|c|c|c|}
\hline Packet size & MSMD OSPF & MSMD OSPA & MSMD OSPAK \\
\hline 200 & 174878 & 27004 & 21226 \\
\hline 400 & 75252 & 6012 & 232 \\
\hline 600 & 45844 & 1252 & 28 \\
\hline 1000 & 24798 & 20 & 16 \\
\hline 1200 & 20004 & 20 & 16 \\
\hline
\end{tabular}

Table 13. SSSD Delay

\begin{tabular}{|c|c|c|c|}
\hline Packet size & SSSD OSPF & SSSD OSPA & SSSD OSPAK \\
\hline 200 & 0.0358164 & 0.0356768 & 0.0361071 \\
\hline 400 & 0.0695581 & 0.0677602 & 0.0230904 \\
\hline 600 & 0.10197 & 0.0875923 & 0.00840145 \\
\hline 1000 & 0.154575 & 0.0110872 & 0.00967739 \\
\hline 1200 & 0.109584 & 0.0119905 & 0.0103122 \\
\hline
\end{tabular}

Table 14. MSMD Delay

\begin{tabular}{|c|c|l|c|}
\hline Packet size & MSMD OSPF & MSMD OSPA & MSMD OSPAK \\
\hline 200 & 0.0174187 & 0.0346311 & 0.0349395 \\
\hline 400 & 0.0340748 & 0.0660935 & 0.0211757 \\
\hline 600 & 0.0503179 & 0.0854756 & 0.00630351 \\
\hline 1000 & 0.0796085 & 0.00831809 & 0.00726269 \\
\hline 1200 & 0.0763801 & 0.00899983 & 0.00774009 \\
\hline
\end{tabular}




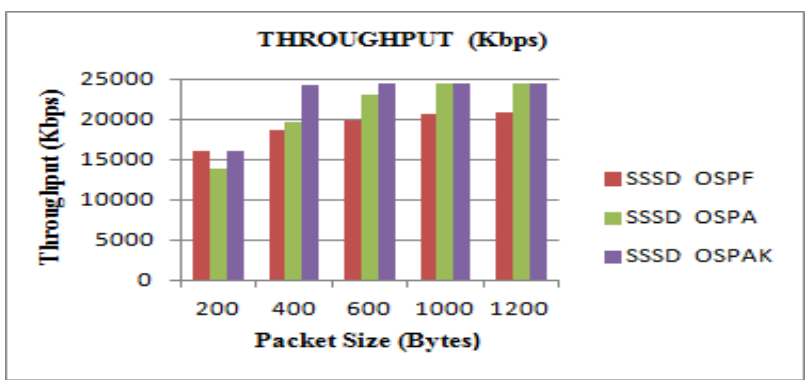

Fig.14. Packet Size vs SSSD Throughput for OSPF, OSPA, OSPAK

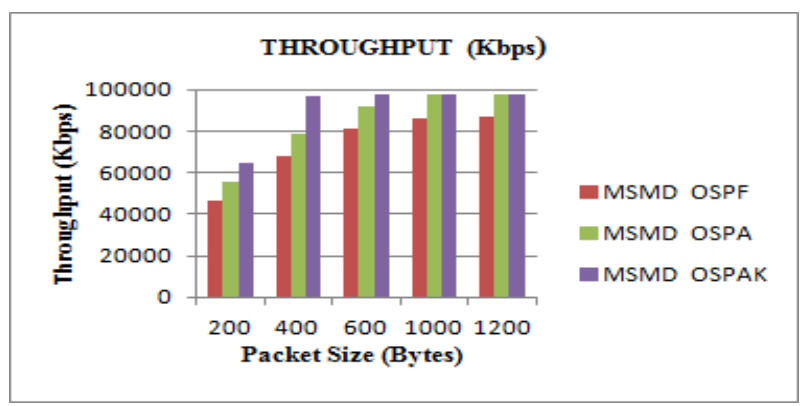

Fig.15. Packet Size vs MSMD Throughput for OSPF, OSPA, OSPAK

Throughput is highest in OSPAK in both SSSD and MSMD scenarios. The higher the number of received bytes of data, the higher is the throughput and that is the reason for higher throughput in OSPAK as shown in Figs. 14 and 15.

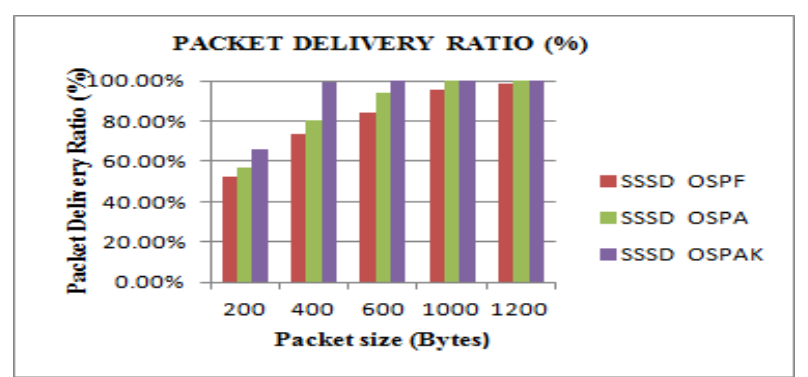

Fig.16. Packet Size vs SSSD Packet Delivery Ratio for OSPF, OSPA, OSPAK

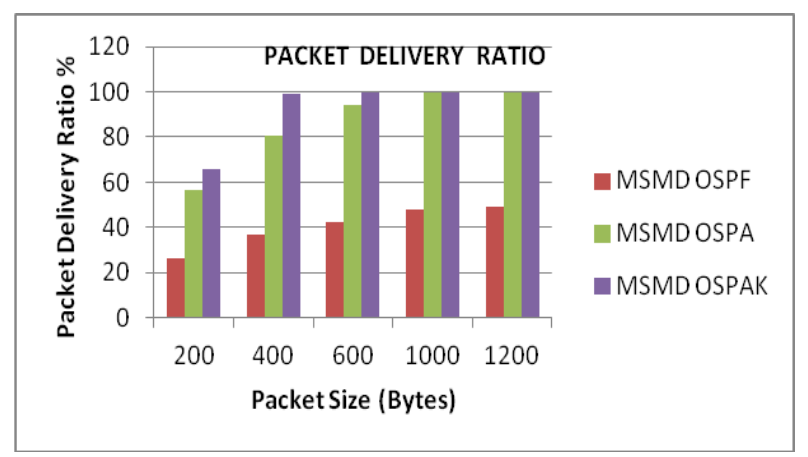

Fig.17. Packet Size vs MSMD Packet Delivery Ratio for OSPF, OSPA, OSPAK.

The Packet Delivery Ratio is highest in OSPAK because of large number of packets being sent and received in OSPAK for both the scenarios SSSD and MSMD as shown in Figs. 16 and 17. 


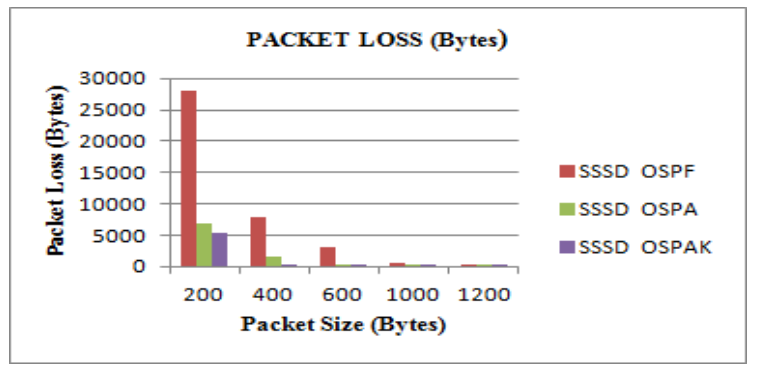

Fig.18. Packet Size vs SSSD Packet Loss for OSPF, OSPA, OSPAK.

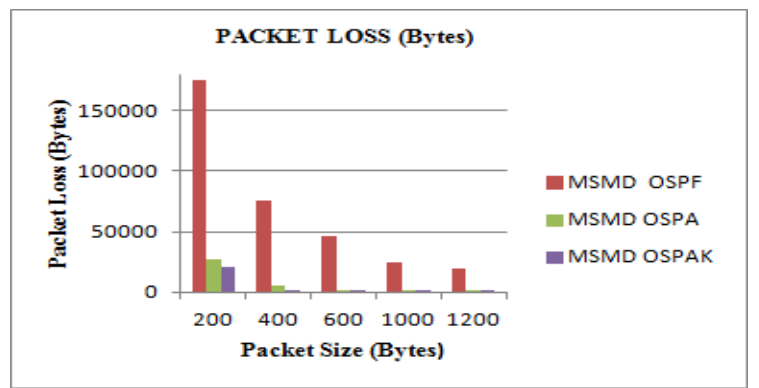

Fig.19. Packet Size vs MSMD Packet Loss for OSPF, OSPA, OSPAK

Throughput is directly proportional to the number of received bytes whileas inversely proportional to packet loss. Therefore Packet Loss is least in OSPAK for both the scenarios SSSD and MSMD as shown in Figs. 18 and 19.

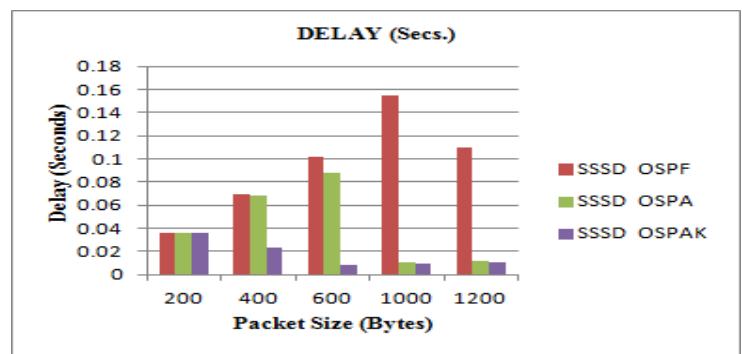

Fig.20. Packet Size vs SSSD Delay for OSPF, OSPA, OSPAK

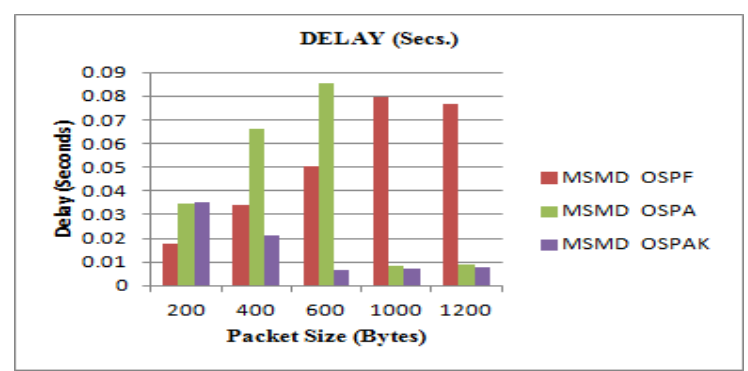

Fig.21. Packet Size vs MSMD Delay for OSPF, OSPA, OSPAK.

Delay is again least in OSPAK because of low reconvergence time as compared to OSPF as shown in Figs. 20 and 21. It is shown in Table 15 and Fig. 22 that as the number of generations increases for each of the destination nodes, the number of $\mathrm{K}$ shortest paths also increases.

Table 15. K Shortest path for each destination node

\begin{tabular}{|c|c|c|c|c|c|c|}
\hline Dest. Node & $\mathrm{N}=500$ & $\mathrm{~N}=1000$ & $\mathrm{~N}=2000$ & $\mathrm{~N}=4000$ & $\mathrm{~N}=6000$ & $\mathrm{~N}=8000$ \\
\hline 1. & 6 & 13 & 14 & 15 & 26 & 36 \\
\hline 2. & 6 & 11 & 13 & 15 & 20 & 22 \\
\hline 3. & 9 & 14 & 15 & 17 & 19 & 23 \\
\hline 4. & 7 & 12 & 14 & 16 & 20 & 22 \\
\hline
\end{tabular}




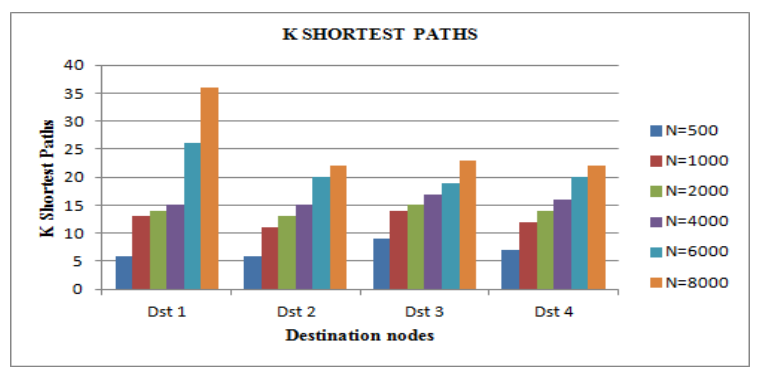

Fig.22. N Generations versus K shortest paths

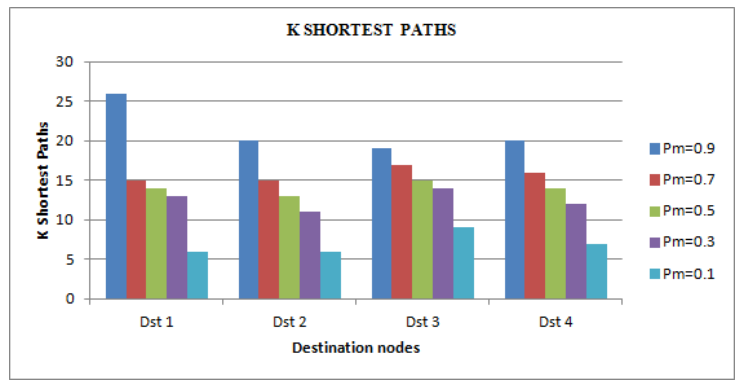

Fig.23. Mutation probability versus K shortest paths

The above figure shows the variation of mutation probability with $\mathrm{K}$ shortest paths. It is seen that as the mutation probability decreases, number of K shortest paths decrease.

\section{Conclusion and Future Scope}

A genetic algorithm is used for the determination of $\mathrm{K}$ shortest paths called K Optimized Shortest path Algorithm (OSPAK), from one single source to more than one destination nodes. Bandwidth addition is not the only necessary option for solving bottlenecks in Networks. Other factors of consideration are Jitter, Packet loss, Delay, Congestion, Buffer overflow. They can affect the performance of Networks. All these parameters have been calculated and compared. A comparison is carried out between Open Shortest Path First(OSPF), Open Shortest Path First with Genetic Algorithm (OSPA) and OSPAK. It is seen from the graphical representation that Throughput and Packet Delivery Ratio is highest when OSPAK is used but OSPA gives lesser Packet loss and Delay. Usage of OSPA or OSPAK will therefore be Application specific, meaning thereby that it depends on the users priority with respect to Packet Delivery Ratio and Delay.Further two more scenarios SSSD and MSMD are taken and it is shown that QoS parameters, namely Throughput and Packet Delivery Ratio are highest in OSPAK and Packet loss and Delay are the least in OSPAK. It is also seen that as the number of generations increases for each of the destination nodes, the number of $\mathrm{K}$ shortest paths also increases. It is also observed that as the mutation probability decreases, the number of $\mathrm{K}$ shortest paths decrease. Effectiveness of the Algorithm OSPAK over conventional algorithms is the reason for it to be used in future. Conventional / Traditional Algorithms compare every possibility to find the best solution versus constraint bound solution by using OSPAK. Furthur, investigations can also be done to reduce the chromosome length in case of networks with a higher number of nodes. The performance can further be improved by, mapping, geographic information with information of destination by taking into account route selection at various stages. Improvements can also be carried out in terms of better crossover and mutation probabilities. The Algorithm OSPA can be used in the future for high speed networks. The effect in relation between population size and size of the network, on the performance of the solution, can be analyzed. Effect of Mutation and Crossover for different population sizes and different topologies can be investigated in the future.

\section{References}

[1] Ahmed, Y. and Hassan, M., 'A genetic algorithm to solve the minimum-cost paths tree problem', In International Journal of Computer Networks \& Communications (IJCNC,), Vol.7, No.4, 2015, pp 75-85.

[2] Ahmed,Y., 'A genetic algorithm for finding the K shortest paths in a network', In Egyptians informatics Journal Vol. 11, 2010, pp 75-79.

[3] Bhasin, H., and Gupta, N., 'Critical Path Problem for Scheduling using Genetic Algorithm',In Conference proceedings on Advances in Intelligent system and Computing, Springer Nature Singapore Pte Ltd., 2018, pp 15-25.

[4] Cheng,H., and Yang, S. 'Genetic Algorithms with Elitism-based Immigrants for Dynamic Shortest Path Problem in Mobile AdHoc Networks', In IEEE Congress on Evolutionary Computation, 2009, pp 3135-3145.

[5] Chowdhury,S., Das,S., and Das,A., 'Application of Genetic Algorithm in Communication Network Security', In International Journal of Innovative Research in Computer and Communication Engineering, Vol. 3, No. 1, 2015, pp 274-280. 
[6] Fadil, Y. 'Routing using Genetic Algorithm for Large Networks', In Diyala Journal of Engineering Sciences, Vol. 3, 2010, pp53-70.

[7] Fall, K. and Varadhan, K. 'The ns Manual: The VINT Project between researchers at UC Berkeley, LBL, USC/ISI, and Xerox PARC', 2011, pp 1-434.

[8] Ghazal, M., Sayed, A., and Kelash, H., 'Routing Optimization using Genetic Algorithm in Ad Hoc Networks', In IEEE Int. Symposium on Signal Processing and Information Technology, 2007, pp 497-503.

[9] Gonen, B., and Yuksel, Y., 'Genetic Algorithm Finding the Shortest Path in Networks', In International Conference on Genetic and Evolutionary Methods in Deptt. Of CSE, University of Nevada, Reno,USA,2011, pp 63-73.

[10] Greg, S., Marie, J.,and Sandra, U., 'Adaptations of k-shortest path algorithms for transportation networks', In Industrial Engineering and Systems Management International Conference, 2015, pp 21-23.

[11] Heidari, A., and Delavar, M., 'A modified Genetic Algorithm for finding fuzzy shortest paths in uncertain Networks', In the International Archives of the Photogrammetry, Remote Sensing and Spatial Information Sciences, Vol. XLI-B2, XXIII ISPRS Congress, , Prague, Czech Republic, 2016, pp 12-19.

[12] Huiping, L, Cheqing J, and Bin, Y, 'Finding Top-k Shortest Paths with Diversity, In IEEE Transactions on Knowledge and Data Engineering', Vol. 30, Issue: 3, 2018, pp 3-14.

[13] Jang, P., Quan, C., and Yeh, C., 'Efficient unicast routing algorithms in Software- Defined Networking', In European Conference on Networks and Communication, 2016, pp 27-30.

[14] Jianyuan, G and Limin. J, 'A new algorithm for finding the $\mathrm{K}$ shortest paths in a time-schedule network with constraints on arcs', In Journal of Algorithms and Computational Technology,Vol.11, Issue 2, 2017, pp 70-77.

[15] Kairanbay, M.,and Hajar, M., 'A Review and Evaluations of Shortest Path Algorithms', In International Journal of Scientific \& Technology Research, Vol. 2, Issue 6, 2013, pp 99-104.

[16] Kavitha S. and Nair T., 'A Comparative Analysis for Determining the Optimal Path using PSO and GA', In International Journal of Computer Applications (0975-8887) Vol. 32, No.4, 2011, pp 8-12.

[17] Keivan, B., and Vahid, H., 'An improved genetic algorithm with a local optimization strategy and an extra mutation level for solving traveling salesman problem', In International Journal of Computer Science, Engineering and Information Technology (IJCSEIT), Vol. 4, Issue 4, 2014, pp 47-53.

[18] Kini, S., Ramasubramanian, S., Kvalbein, A. and Hansen, A., 'Fast Recovery from Dual-Link Failures in IP Networks', In Proceedings of IEEE International Conference on Computer Communications (INFOCOM), Rio de Janeiro, Brazil, 2009, pp 1368-1376.

[19] Kumar, N., Misra, S., Obaidat, M., 'Collaborative Learning Automata-Based Routing for Rescue Operations in Dense Urban Regions Using Vehicular Sensor Networks', In IEEE Systems Journal, DOI: 10.1109/JSYST.2014 22335451, 2014, pp 10811090.

[20] Agarwal, U., and Gupta, V., 'Network Routing Algorithm using Genetic Algorithm and Compare with Route Guidance Algorithm', In International Journal of Scientific Research Engineering and Technology, Conference proceedings IEERET, , 2014, pp 92-96.

[21] Kumar, R. and Kumar, M. 'Exploring GA for Shortest Path Optimization in Data Networks', In Global Journal of Computer Science and Technology, Vol 10, No. 11, 2010, pp 8-13.

[22] Kumari, S. and Geethanjali, N., 'A Survey on Shortest Path Routing Algorithms for Public Transport Travel”, In Global Journal of Computer Science and Technology, Vol. 9 Issue 5 ,Ver 5., 2016, pp 73-77.

[23] Misra, S., 'An Adaptive Online Routing Algorithm for MPLS Traffic Engineering', In Proceedings of the 3rd International Conference for Upcoming Engineers (ICUE2004), IEEE Toronto, Ontario, 2004,pp 959-976.

[24] Misra, S., and Oommen, B., 'Adaptive Algorithms for Network Routing and Traffic Engineering', In Proceedings of the 19th National Conference on Artificial Intelligence,SanJose,California,USA, 2004.

[25] Misra, S., and Rajesh, G., 'Bird Flight-Inspired Routing Protocol for Mobile Ad Hoc Networks', In ACM Transactions on Autonomous and Adaptive Systems, Vol. 6, No. 4, 2011, pp 843-852.

[26] Misra, S., Ghosh, T., Obaidat, M., 'Routing Bandwidth Guaranteed Paths for Traffic Engineering in WiMAX Mesh Networks', In International Journal of Communication Systems(Wiley),Vol.27, No.11, 2012, pp. 2964-2984.

[27] Misra,S., Krishna, P., Bhiwal, A., Chawla, A., Wolfinger, B., Lee, C., 'A Learning Automata-Based Fault-Tolerant Routing Algorithm for Mobile Ad Hoc Networks', In the Journal of Supercomputing (Springer), Vol. 62, No. 1, 2012, pp. 4-23.

[28] Moza, M., and Kumar, S., , 2018, 'Routing in networks using genetic algorithm', In International Journal of Communication Networks and Distributed Systems, Vol. 20, No. 3, 2018, pp. 291-311.

[29] Obeidat, A .F. and Alshalabi, M. E., 'Improving the Performance of the Networks Using Genetic Algorithm', In Proceedings of the International Conference on Advances in Computer and Information Technology, 2012, pp 33-37.

[30] Qingson, W., Ren, C., Lifeng, N., and Yinglong, X., ' Finding Top K Shortest Simple Paths with Improved Space Efficiency', In ACM, Vol 9, Issue 5, 2017, pp 1-6.

[31] Reza, Roshani., and Mohammad, K., 'Parallel Genetic Algorithm for Shortest Path Routing Problem with Collaborative Neighbors',In Ciência eNatura, Vol. 3,Issue 2, 2015, pp. 328-333.

[32] Sonam, J., and Sandeep, S., 'The Application of Genetic Algorithm in the Design of Routing Protocols in MANETs: A Survey', In International Journal of Computer Science and Information Technologies, Vol. 3, No. 3, 2012, pp $4318-4321$.

[33] Theodoros, C., and Panagiotis, B., 'Exact and Approximate Algorithms for Finding k-Shortest Paths with Limited Overlap', In 20th International Conference on Extending Database Technology (EDBT), Venice, Italy, 2017, pp 414-425.

[34] Umit, A., Ismail, R., Cevdet, G., Beyza, Y., and Ilhami, M., 'An Idea for Finding the Shortest Driving Time Using Genetic Algorithm Based Routing Approach on Mobile Devices', In International Journal of Mathematics and Computers in Simulation, Vol. 6 , Issue 1, 2012, pp 9-16.

[35] Yang,S., Cheng, H., and Wang,F., 'Genetic Algorithms With Immigrants and Memory Schemes for Dynamic Shortest Path Routing Problems in Mobile Ad Hoc Network', In IEEE transaction on System, Man, and Cybernetics-Part C: Application and Reviews, Vol 40, No. 1, 2010, pp 52-63. 
[36] Yusof, R., Khairuddin, U., and Khalid, M., 'A New Mutation Operation for Faster Convergence in Genetic Algorithm Feature Selection',In International Journal of Innovative Computing, Information and Control, Vol. 18, No. 10, 2012, pp 7363-7380.

\section{Authors' Profiles}

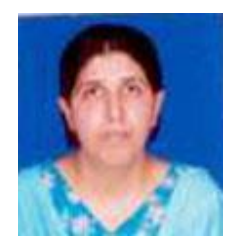

Dr. Meenakshi moza born in Srinagar on 25th Jan 1965, did her B.E from R.E.C. Srinagar (Kashmir) in Electronics and Communication. She completed her Mtech from Y.M.C.A Faridabad. She has done Phd in the field of computer networks. She has 19 years of experience in teaching and 8 years of experience in industry namely Onida, Avery India. Total number of research publications are 20.

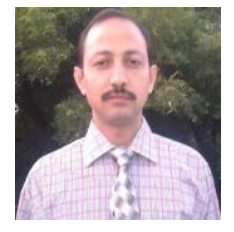

Dr. Suresh Kumar is a professor in MRIIRS. His qualifications are as mentioned. Ph.D.(Computer Science \& Engg.),UGC NET(Computer Science \& Engg), M.Tech.( Computer Science \& Engg.), B.Tech. (Computer Science \& Engg.). He has 18 years of teaching experience and his areas of interest include Networking, Operating systems, Database management system. Total number of research publications are 31.

How to cite this paper: Meenakshi Moza, Suresh Kumar, "Finding K Shortest Paths in a Network Using Genetic Algorithm", International Journal of Computer Network and Information Security(IJCNIS), Vol.12, No.5, pp.56-73, 2020. DOI: 10.5815/ijcnis.2020.05.05 